Rev. Elev. Méd. vét. Pays trop., 1973, 26 (4) : 5 a - 11 a

\title{
Les conserves de poissons au Sénégal
}

\author{
par J. ROZIER $\left(^{*}\right)$ et J. CHANTAL $\left(^{*}\right)$
}

\begin{abstract}
RESUME
La production totale de poissons au Sénégal est importante (220000 tonnes en 1971). La pêche industrielle s'est développée depuis 10 ans environ. Trois grosses conserveries: l'usine des a Conserveries du Sénégal $»$, celle de la S.A.P.A.L. et celle de la S.A.I.B., traitent le thon et les sardinelles. Les conserves, correspondant à environ 30000 tonnes de poissons frais, sont de très bonne qualité.

Un service de contrôle assure une surveillance permanente depuis la pêche jusqu'à la commercialisation. La plus grande partie des conserves est expédiée vers l'Europe.
\end{abstract}

En ce qui concerne la pêche, tant artisanale qu'industrielle, le Sénégal est remarquablement situé. La presqu'île du Cap Vert, aérée et de climat toujours relativement frais, offre un refuge idéal pour les gros bateaux de pêche. Le port de Dakar est particulièrement apte à recevoir une structure industrielle de conserveries de poissons alors que la mer, au large des $700 \mathrm{~km}$ de côtes, est l'une des plus riches du monde en raison des courants marins qui y existent. La production de matières organiques y est d'environ $0,70 \mathrm{~g} \mathrm{par} \mathrm{m}^{2}$ par jour alors que, plus au nord, au niveau des Caraïbes, elle n'est plus que de $0,03 \mathrm{~g} \mathrm{par} \mathrm{m}^{2}$ par jour. Cette prolifération du plancton permet le développement de 910 espèces de poissons habituellement consommés entre le Maroc et la Guinée.

Parmi toutes ces espèces, quelques-unes seulement peuvent être utilisées de manière industrielle. Certains Thonidés et Clupéidés (Sardina et Sardinella) sont d'ailleurs exploitées depuis plusieurs années par de nombreux pays, non seulement africains mais aussi extra-africains. On peut considérer que l'Atlantique intertropicale est le siège de toutes les flottes thonières du monde. Mais déjà cette exploitation intensive pose le problème de conservation des stocks.

Le Sénégal a compris depuis longtemps les avantages qu'il pouvait tirer d'une telle situation. En 1971, avec une production globale de poissons de 220000 tonnes, en augmentation de 30 p. 100 par rapport à 1970 , il était le $4^{\mathrm{e}}$ pays producteur d'Afrique, après l'Afrique du Sud, l'Angola et le Maroc. Le tableau ci-dessous donne l'origine de cette production de 1969 à 1971 exprimée en milliers de tonnes (4).

\begin{tabular}{|c|c|c|c|c|c|}
\hline & $\begin{array}{c}\text { Pêche } \\
\text { artisanale }\end{array}$ & Sardiniers & Chalutiers & Thoniers & Total \\
\hline 1960 & 80 & & & & \\
\hline 1969 & 126 & 18 & 6 & 12 & 162 \\
\hline 1970 & 133 & 15 & 9 & 12 & 170 \\
\hline 1971 & 179 & 13 & 9 & 18 & 220 \\
\hline
\end{tabular}

(*) Professeurs à l'Ecole Inter-Etats des Sciences et Médecine Vétérinaires, Dakar - Sénégal. 
Devant l'envahissement de la mer par des bateaux étrangers, le Sénégal a été amené à protéger ses zones de pêches. En février 1970, la limite des eaux territoriales était portée à 12 milles et en février 1972 l'extension des zones de pêche atteignait 110 milles. Ces zones ne sont accessibles qu'aux bateaux sénégalais, gambiens ou français possédant une licence.

La pêche thonière est assurée par les bateaux sénégalais de la SOSAP (Société Sénégalaise d'Armement à la Pêche) qui possède 19 unités; ils ont débarqué 11104 tonnes en 1971. La flotte bascobretonne, 30 unités, a de son côté débarqué (à Dakar) 7357 tonnes la même année. Ces prises sont aussitôt réparties entre les conserveries selon des quotas fixés par le Gouvernement.

Les conserveries de sardinelles disposent de leur côté d'apports importants fournis par la pêche industrielle et la pêche artisanale.

Le Sénégal possède aussi des usines de surgélation de poissons et de crustacés. Nous ne parlerons pas de cette production aux problèmes particuliers, limitant notre sujet aux conserves appertisées.

Après avoir examiné les conserveries et leurs productions, nous envisagerons le contrôle de la fabrication et des produits finis qui garantit la qualité des conserves.

\section{LES CONSERVERIES DE POISSONS AU SENEGAL}

Ce secteur de l'industrie alimentaire a évolué dans le sens d'une concentration depuis les années 60. A l'heure actuelle il existe trois usines : celle des "Conserveries du Sénégal», celle de la "Société Africaine de Produits Alimentaires» (S.A.P.A.L.) et celle créée par la «Société Africaine des Industries du Bâtiment »(S.A.I.B.).

\section{A, L'Usine des “ Conserveries du Sénégal "}

Cet établissement, situé sur le port de pêche de Dakar, a été inauguré le 7 novembre 1968. Cette très belle réalisation de $15000 \mathrm{~m}^{2}$ couverts résulte de l'exécution d'un protocole d'accord entre le Gouvernement sénégalais et la Société Coopérative "Pêcheurs de France » désignée comme maître d'œuvre.

Sa capacité de production annuelle est de 15000 à 18000 tonnes de thons et de 7000 tonnes de sardinelles. La Direction des pêches lui attribue $62 \mathrm{p}$. cent des thons débarqués. Son activité la range au $11^{\mathrm{e}}$ rang des industries sénégalaises avec un chiffre d'affaires de 1742 millions de francs C.F.A. en 1970. Elle a employé, en 1971, 503 ouvriers permanents et 400 journaliers, ce qui représente une masse salariale d'environ 68 millions C.F.A. Sa production comporte du thon au naturel, du thon à l'huile et à la tomate, des sardinelles à l'huile entières ou en filet.

L'usine, située à quelques mètres du quai de débarquement, possède 3 chambres de congélation et de stockage du poisson à $-20^{\circ} \mathrm{C}$ d'une capacité totale de 1500 tonnes. Ces installations assurent un fonctionnement continu malgré les arrivées irrégulières. L'établissement comprend quatre lignes de fabrication de conserve au naturel, cinq chaînes de thon cuit et trois chaînes de traitement des sardinelles. L'emboîtage, manuel ou mécanique pour certaines productions, précède la stérilisation assurée par une batterie de 33 autoclaves cylindriques verticaux. Un grand local permet le stockage de trois semaines de production.

L'usine traite aussi les sous-produits dans une installation automatisée Alfa-Laval. Elle a fabriqué, au cours de la campagne de pêche 1971-72, 1000 tonnes de farine de poisson qui ont été exportées vers l'Europe et 40000 litres d'huile utilisés par la « Société Electrique et Industrielle du Baol »(S.E.I.B.) à Diourbel dans la fabrication de margarine et d'huile de table. 
Cette récupération rationnelle des sous-produits présente un gros intérêt pour l'économie nationale et devrait trouver, quant aux farines, un débouché local dans le cadre de l'embouche bovine.

\section{B. L'Usine de la S.A.P.A.L.}

En 1960, le groupe Saupiquet-Graciet absorbait dans son établissement les 7 unités existant alors. Aujourd'hui, à la suite de la modernisation de ses équipements, l'usine a une capacité annuelle de 12000 tonnes. Le quota de thon qui lui est attribué est de 38 p. cent. Elle a employé en 1971, 420 ouvriers permanents et 110 ouvriers journaliers, ce qui a représenté une masse salariale de 35 millions C.F.A. Elle se situait au $21^{\mathrm{e}}$ rang des industries sénégalaises en 1970 par son chiffre d'affaires de 795 millions de francs C.F.A. L'usine de la S.A.P.A.L. ne traite que le thon. Les installations de traitement sont comparables à celles de l'usine des « Conserveries du Sénégal » mais disposées d’une façon plus ramassée.

\section{L'Usine de la S.A.I.B.}

Elle ne représente qu'un secteur de cette société classée au $30^{\circ}$ rang des industries sénégalaises en 1970 par son chiffre d'affaires de 570 millions de francs C.F.A. Créée en mai 1966, sa capacité annuelle est de 5000 tonnes. Elle ne traite que des sardinelles pêchées par des chalutiers, des sardiniers ou au filet tournant par les piroguiers, surtout ceux de Hann, Bel Air, Thiaroye et M'Bao. Elle traitera le thon dès janvier 1974.

L'usine a traité 1300 tonnes de poissons en 1971. Elle travaille donc, à l'heure actuelle, en dessous de sa capacité, faute d'arrivage. En 1972, elle employait 120 ouvriers dont 100 femmes. Les conserves fabriquées sont les sardinelles à l'huile pimentées ou non, à la tomate, les filets de sardinelles à l'huile, les pilchards à la tomate et les pâtes de sardinelles. Les deux chaînes de travail du poisson précèdent des installations mécaniques modernes de jutage, huilage, sertissage. Les autoclaves sont de type vertical.

Ainsi le Sénégal dispose de trois établissements bien équipés, capables de traiter de façon rationnelle le fruit de sa pêche et de valoriser ces matières premières. Examinons sommairement les diverses fabrications.

\section{LES DIVERS TYPES DE CONSERVES ET LEUR COMMERCIALISATION}

Il convient d'envisager les deux groupes, thonidés et clupéidés, de façon séparée en raison de leur traitement différent imposé par leur qualité respective.

\section{a) Les thons}

Il existe plusieurs espèces de thons dont la destination est fonction de leur qualité. L'《 albacore » sert à la fabrication du thon au naturel, le « listao », du thon cuit à l'huile ou à la tomate. Le "patudo " est parfois utilisé dans l'une ou l'autre production selon qu'il pèse moins ou plus de $35 \mathrm{~kg}$.

Après réception et contrôle de leur qualité technologique, les poissons sont dirigés soit vers la congélation, soit vers les chaînes de fabrications. Dans ce dernier cas, il se passe moins de deux heures entre le débarquement et l'emboîtage. Les thons n'auront donc pas à souffrir de la chaleur des tropiques.

Pour le thon au naturel, les "albacores" $(20$ à $30 \mathrm{~kg}$ ) sont tronçonnés selon l'épaisseur voulue pour l'emboîtage. Les tranches sont parées. Les ouvrières retirent les arêtes et les muscles bruns. Les morceaux sont alors saumurés, entraînés dans une longue cuve de saumure par une vis sans fin dont la vitesse de rotation règle le temps d'immersion. La durée de salage est fixée par la nature du poisson et son état frais ou décongelé. Un rinçage à l'eau douce précède l'emboî- 
tage manuel dans des formats variables selon la demande. Le poids des boîtes est vérifié avant jutage à chaud avec une saumure très douce, légèrement aromatisée. Les opérations suivantes demeurent classiques : sertissage et autoclavage à $115^{\circ} \mathrm{C}$.

Pour le thon cuit, les " listao" sont étêtés, éviscerés et disposés dans de grands casiers métalliques perforés pour être cuits en saumure, aromatisés. Après cuisson les poissons sont mis à refroidir à l'air pendant douze heures, ce qui assure leur séchage. Les thons sont alors parés par élimination de la peau, des arêtes, des muscles bruns profonds et des parties superficielles qui ont bruni pendant le séchage. Cette opération est délicate, et c'est à ce stade que peuvent se produire des pertes qui diminuent le rendement. Les morceaux de muscles cuits sont pesés et introduits dans des moules cylindriques où ils sont comprimés à l'aide d'un piston. Ainsi remplis, les moules sont posés sur des emboiteuses automatiques qui tranchent à l'épaisseur voulue ce rouleau pressé et poussé progressivement. Néanmoins les gros boîtages $1 / 1$ et $3 / 1$ sont remplis à la main. Le poids est alors contrôlé. Les boîtes reçoivent l'huile, sont serties et lavées avant d'être autoclavées. L'adjonction de concentré de tomate est généralement réservée aux boîtes remplies de petits morceaux.

Les conserves ainsi fabriquées sont conditionnées en cartons après vérification, stockées sur palettes et revérifiées au moment de l'expédition pour déceler les fuitages et les bombages éventuels. On considère que 1 tonne de poisson frais permet de fabriquer $700 \mathrm{~kg}$ de conserve, boîtes comprises. Les déchets sont donc de l'ordre de 30 à 40 p. cent.

Les débouchés des conserves de thons sont à 98 p. 100 extra-sénégalais, car les sénégalais ne consomment pas les thonidés. L'explication suivante est évoquée :

L'altération des thons conservés dans de mauvaises conditions, se traduit par l'apparition précoce d'histamine. Il est vraisemblable que les sénégalais ayant observé depuis fort longtemps les effets désagréables de l'histamine à la suite de la consommation de ces espèces de poisson, les aient considérés comme non consommables.

Le principal débouché est la France: Des accords franco-sénégalais définissent chaque année le contingent de conserves sénégalaises admises en franchise de droit à l'importation en France : 12500 tonnes actuellement. Une faible proportion de boîtes est dirigée vers d'autres pays européens, américains ou soviétiques.

\section{b) Les sardinelles}

Très abondantes, les sardinelles arrivent fraîches à l'usine. Après réception et élimination des lots défectueux, les poissons sont étêtés, écaillés, éviscerés. Ces opérations sont, pour la plupart, effectuées manuellement. Les petites sardinelles sont introduites crues dans les boîtes, cuites à la vapeur dans la boîte ouverte, égouttées, couvertes d'huile additionnée d'épices et de condiments. Les boîtes sont alors serties et autoclavées après lavage. Les sardinelles de plus grosse taille sont cuites à la vapeur, les filets sont levés, introduits dans des boîtes, recouverts d'huile ou de concentré de tomate.

Enfin pour certains poissons de qualité technologique moindre, à la cuisson fait suite un parage, un broyage et un malaxage avec addition d'huile. La pâte obtenue est introduite dans des boîtes cylindriques.

A la différence des conserves de thon, les conserves de sardinelles trouvent des débouchés au Sénégal et en Afrique. Ces poissons y sont consommés depuis longtemps, les conserves répondent aux goûts des habitants. Notons aussi que leur prix modique leur ouvre une clientèle assez large. La majeure partie des pilchards est exportée sur l'Europe. Pour ces raisons, ce secteur de l'industrie de la conserve du poisson doit être développé et soutenu, le cas échéant.

Ce domaine de l'industrie alimentaire, déjà remarquable par le tonnage traité et la diversité de la production, s'impose par la qualité des produits fabriqués dont le contrôle permanent est un des soucis premiers de la Direction des pêches et des industriels eux-mêmes. 


\section{CONTROLE DE LA QUALITE DES CONSERVES DE POISSONS}

Deux types de contrôles cuvrent dans le même sens du maintien de la qualité et éventuellement de son amélioration.

Le premier est réalisé par les industriels eux-mêmes. Ses modalités dépendent de l'initiative privée. La seule sanction est commerciale. L'intérêt évident des fabricants étant de fournir à leur client une "bonne" marchandise de qualité constante.

Le second est officiel. Il dépend du Service des Pêches, dans le cadre du Ministère du Développement rural. Une brigade, composée de 6 agents techniques ayant reçu une formation spécialisée, placée sous la responsabilité d'un vétérinaire, assure le contrôle permanent depuis la réception des poissons jusqu'à la commercialisation des boîtes. La compétence de ces agents et la conscience qu'ils manifestent dans l'accomplissement de leur tâche, les fait travailler en collaboration confiante avec le personnel de l'usine.

Ce contrôle comporte plusieurs étapes : La première est celle de l'inspection des matières premières.

L'examen des lots de thons est réalisé dès le débarquement qui a été annoncé par radio. Au cours de cette inspection macroscopique, un faible pourcentage de poissons altérés est saisi: moins de 5 p. 100 pour les lots frais, moins de 2 p. 100 pour les lots congelés. En 1972, il n'a été saisi que 5 tonnes. En général il s'agit de poissons écrasés ou abîmés. En période très chaude, on peut observer parfois un début d'altération microbienne. Les poissons saisis sont transformés en farine. Le Service des Pêches, ayant agréé la marchandise, délivre un certificat de salubrité. Il est responsable également de la répartition en fonction des quotas.

Ce contrôle systématique, rigoureux est effectué de la même façon pour les sardinelles. En raison des origines variables de ces espèces, sardiniers, piroguiers, il arrive que certains lots ne soient pas entièrement satisfaisants. Ils sont écartés de la fabrication dès qu'est décelée la moindre altération.

Huile, concentré de tomate, sel, etc., sont également contrôlés à la réception.

Le contrôle se poursuit tout au long de la fabrication. Les responsables des chaînes doivent veiller sans arrêt à ce que les pertes dues au parage soient minimales et, en même temps, à ce que le parage soit correctement fait. Nous avons vu ci-dessus que ce stade de préparation était le plus délicat.

Par ailleurs le Service des pêches, responsable de l'hygiène, est constamment tenu en éveil. Il s'agit de former et d'informer le personnel. Les installations sanitaires convenables sont utilisées comme il le faut. Il existe un apprentissage de cet usage et les agents de la brigade d'inspection doivent montrer beaucoup de patience et faire preuve d'indulgence. Ils savent bien que leur mission n'est pas répressive mais éducatrice. Ils sont aidés dans leur travail par les chefs d'équipe. L'examen des fiches d'enregistrement des termpératures d'appertisation permet de vérifier si les barèmes de stérilisation ont été convenablement appliqués. Enfin, après autoclavage chaque boîte est manipulée et vérifiée par les ouvriers quí assurent l'emballage.

Le contrôle du produit fini s'effectue de plusieurs façons: Dans la plupart des cas les normes techniques sont vérifiées par les laboratoires des usines. Le degré de remplissage (poids égoutté), la qualité des poissons, la qualité des ingrédients sont appréciés. Ces mesures et analyses rentrent aussi dans le cadre des prérogatives du Service de la Répression des Fraudes. Le Service des Pêches prélève systématiquement, dans chaque lot de fabrication, un certain nombre de boîtes ( 7 en moyenne) qui sont soumises à diverses analyses de laboratoire. Ce travail de bactériologie et de biochimie est réalisé dans les locaux de l'Institut de Technologie Alimentaire (I.T.A.) et du Centre Océanographique de Thiaroye. Généralement les épreuves effectuées sont l'étuvage pour rechercher les défauts d'appertisation et les dosages d'A.B.V.T., d'histamine et d'indole pour apprécier le degré de fraîcheur des poissons mis en cuvre. Les résultats sont 
pratiquement toujours satisfaisants. Des dosages effectués en France par nos soins sur des boîtes de thon fabriquées au Sénégal ont montré que les taux d'histamine sont inférieurs à 5 p.p.m., le plus souvent les taux sont si faibles qu'ils n'atteignent pas le degré de sensibilité de la méthode biologique utilisée (action d'extraits aqueux sur un iléon de cobaye en liquide de thirode).

A côté de ce contrôle classique traditionnel, signalons que les locaux d'entreposage des boîtes de conserves ont des températures voisines de $30^{\circ} \mathrm{C}$, souvent supérieures, ce qui correspond à celle d'une étuve d'incubation bactériologique. Autrement dit, ce n'est pas seulement un échantillonnage plus ou moins représentatif qui est soumis au contrôle, mais toute la fabrication. Au moment de l'expédition, les boîtes bombées ou fuitées sont éliminées. On recherchera l'origine de l'altération. Dans l'ensemble, le nombre de boites défectueuses est inférieur à 1 pour 1000 . On sait que ce chiffre correspond à un taux normal de défectuosités inévitables dans le domaine de la conserverie.

Cette description du contrôle souligne avec quelle application les services officiels assurent leur tâche pour maintenir une bonne qualité aux productions de ce secteur de l'industrie alimentaire, avant d'accorder le permis d'exportation.

\section{CONCLUSION}

Cette présentation succincte des conserves de poissons aura montré, nous l'espérons, le dynamisme de cette industrie, en développement constant depuis dix ans. De nombreux efforts sont tentés sur le plan social et la stabililité de la main-d'œuvre est une preuve de leur réussite.

Sur le plan international le Sénégal, par le modernisme de ses installations, par l'importance de ses structures, peut espérer accroître sa production. La qualité des conserves lui donne une place privilégiée dans la concurrence avec les pays étrangers.

Cette situation se traduit par de nombreux projets dont certains sont en cours de réalisation. Des investissements sont prévus pour l'armement thonier, pour l'achat de cargos frigorifiques, pour la création d'un complexe sardinier et l'installation d'entrepôts frigorifiques et de nouveaux ateliers de transformation.

Il ne faut pas oublier par ailleurs la production de produits de la mer congelés et surgelés : crevettes, langoustes dont le développement est parallèle.

Si l'expansion de la pêche maritime a été l'un des faits marquants de l'économie sénégalaise au cours des années 1960 , cette expansion se poursuit à vive allure depuis 1970 et devrait continuer par la suite tant en importance qu'en diversification des productions de luxe comme de consommation courante.

\section{REMERCIEMENTS}

Nous tenons à remercier, pour l'accueil qu'ils nous ont ménagé et les renseignements qu'ils nous ont fournis

MM. les Docteurs vétérinaires :

I. M. DIA, Directeur de l'Océanographie et des Pêches maritimes du Sénégal;

B. DIOP, Directeur Général de la SOSAP

et MM.

OLLIVIER, Directeur de l'usine des Conserveries du Sénégal;

BALLEREAU, Directeur Adjoint de l'usine de la SAPAL;

C. SCHEFFER, Directeur de la S.A.I.B.

Mme CHERMETTE, Directrice du Département Conserverie de la S.A.I.B. 


\section{SUMMARY}

\section{The preserve of fishes in Senegal}

The total production of fish is important in Senegal (220000 tons in 1971). The industrial fishing has been expanding for ten years about. There are three big preserving: the factory of "Conserveries du Sénégal ", the one of the S.A.P.A.L. and the other one of the S.A.I.B., which treat the tunny-fish and the sardinelle.

The preserves, 30000 tons equivalent fresh fish, are a very good quality. A control department assures a permanent supervision from fishing to commercialization. The biggest part of preserves is exported towards Europe.

\section{RESUMEN}

\section{Conservas de pescados en Senegal}

La producción total de pescados en Senegal es importante (220 000 toneladas en 1971). La pesca industrial se ha desarrollado desde unos diez años. Tres fábricas de conservas: la de Senegal, la de la «S.A.P.A.L. » y la de la «S.A.I.B. » tratan los atunes y las sardinetas. Las conservas. representando unas 30000 toneladas de peces, son de muy buena calidad. Un servicio de control atende una vigilancia permanente desde la pesca hasta la mercantilización. Se envia la más grande parte de las conservas hacia Europa.

\section{BIBLIOGRAPHIE}

1. DUTRIEUX (L.). Les a Conserveries du Sénégal », un pari sur l'avenir en bonne voie d'être gagné. Rev. Conserve, 1969 (9) : 99-107.

2. L'Economie sénégalaise. 1re éd. Paris, Ediafric (57, avenue d'Iéna, XVIø). 1970. Bull. Afr. noire, numéro spécial.

3. Enquête sur les ressources naturelles du continent africain. Paris, UNESCO, 1963.

4. Mémento de l'économie africaine. $7 \mathrm{e}$ éd. Paris, Ediafric, 1972. Bull. Afr. noire, numéro spécial. 